literature relating neuroanatomic systems and depression. The chapter contains little gems like the inclusion of scales appropriate for the assessment of depression in aphasic patients and is an excellent chapter for a brief review of a complex subject.

The chapter by Dieguez, Staub, and Bogousslavsky concerning asomatognosia was both the most interesting and least scientifically satisfying. It is among the longest chapters, 36 pages, including an appendix entitled "A funneled guideline for the bedside interview". The authors present the reader with a diagnostic entity termed an asomatognosia which they state is "roughly defined as the disturbances of the body schema, (and) encompasses a wide array of clinical pictures under a unitary conceptual framework" (p. 215). The authors note that usually asomatognosia is viewed as a subsection of the study of anosognosia or neglect. However, they propose to return to the French tradition, that of Lhermitte (1939) and Hecaen \& de Ajuriaguerra (1952) in which anosognosia was viewed as an instance of hemiasomatognosia. The authors then present a very detailed and well reasoned explication of their framework for disorders of body schema. However, the level of detail contained in the framework far outstripped the known loci of lesions causing each of the detailed phenomena. The reader is left, therefore, with a "think piece" concerning the nature and etiology of disorders of body schema with very little clinicalpathological correlative data to support the reliability and validity of the constructs. Overall, while this chapter offers the reader an impressively logical framework, the level of authoritative literature supporting the validity of the framework was well below the level offered in the other chapters.

In summary, The Behavioral and Cognitive Neurology of Stroke is an excellent source book. It is likely to be re-read many times by its owner; indeed, every time the clinician is requested to see an "interesting" patient and needs a fast review of the phenomenology, assessment procedures, neuroanatomic implication, and appropriate treatment plan for the patient he/she is about to encounter.

\section{REFERENCES}

Lhermitte, J. (1939). L'Image de Notre Corps. Paris: Nouvelle Revue Critique.

Hecaen, H. \& de Ajuriaguerra, J. (1952). Meconnaissances et Hallucinations Corporelles. Integration et disintegration de la somatognosie. Paris: Masson.

\title{
What's New With the Frontal Lobes?
}

DOI: $10.1017 /$ S1355617708080247

\section{The Human Frontal Lobes: Functions and Disorders (Second Edition). 2007. Bruce L. Miller \& Jeffrey L. Cummings (Eds.). New York: The Guilford Press, 666 pp., \$95.00 (HB) \\ Reviewed by Vicki Anderson, Ph.D., Department of Psychology, Royal Children's Hospital, Parkville, Victoria, Australia}

Miller and Cummings published their original text, The Human Frontal Lobes: Functions and Disorders, in 1999. At that time, their work was quite unique and provided a valuable reference for both health professionals and researchers in the emerging field of the frontal lobes. The years since then have seen an increasing focus on these fascinating brain structures across a range of disciplines including neurosciences, neurology, neuropsychology and psychiatry. The importance of the frontal structures for both cognition and emotion is firmly established and the complexity of these associations continues to challenge those working in these fields. As Miller and Cummings note, due to the major advances that have occurred since they first published their work, their second edition represents not only an update but also an introduction to a range of new issues. In particular, this second edition emphasizes advances in neurosciences and neuroimaging with a substantial amount of new material presented in these areas. In addition, the increasing interest in the role of the frontal lobes in social and emotional function is also acknowledged and discussed.

The text is divided into an introduction and six content areas: anatomy, neurochemistry, neuroimaging, neuropsy- chology, neurology and neuropsychiatry. Chapters vary from highly research focused to clinical in nature, but with an underlying emphasis on providing an evidence-base for the reader. The text benefits from the joint neurological and psychiatric expertise of the authors, and there is an attempt throughout to link these two areas, as well as to relate basic science with clinical issues.

The introductory sections of the text illustrate the breadth of knowledge of the editors. They provide a chronological account of the key factors which have facilitated the study of the frontal lobes, starting with the well known tale of Phineas Gage, and moving through a number of influential advances in the field, noting conceptual leaps, such as the emergence of the theory of working memory, as well as the gradual acknowledgment of the importance of the frontal lobes to a wide range of clinical conditions, such as dementias, depression and schizophrenia. The changing nature of the field is highlighted, with the frontal lobes and their functions initially viewed as largely uni-dimensional, but with a more contemporary perspective gradually emerging in keeping with scientific advances, and taking on a more multi-dimensional systemic framework. These chapters also 
incorporate definitional details for the field and provide the reader with some basic information on frontal structures and functions that is extended and elaborated in later chapters.

There are six chapters addressing the anatomy of the frontal lobes, covering topics such as fronto-striatal circuitry, dorsolateral and cingulate cortex, orbitofrontal cortex and the insula, gross morphology and evolutionary issues. Each of these chapters provides a contemporary discussion of these areas, and of note, a number have made an effort to relate anatomical data and clinical syndromes. For example, the chapter addressing fronto-striatal circuits includes a discussion of relevant neuropsychiatric syndromes, including attention deficit hyperactivity disorder, depression and fronto-temporal dementia. A chapter by Geschwind and Iacaboni considers structural and functional asymmetries of the frontal lobes, and the final contribution to this section provides a fascinating account of the evolution of frontal structures.

There are three chapters relating to neurochemistry, illustrating the growth of knowledge in this field over recent years. One of these chapters, describing the mesocortical dopaminergic system, is a new contribution to the text and identifies the importance of neurotransmitters for enhancing knowledge of social and cognitive functions.

The third key area of focus is neuroimaging, and Miller and Cummings identify that this has been an area of particularly rapid growth over the past decade. To address this domain, they have included six chapters, which together provide an excellent coverage of the importance of imaging approaches to re-conceptualizing the functions of the frontal lobes. The initial chapter introduces the reader to the complexities of structural imaging methods, describing a range of image acquisition models as well as data analytic techniques. There is an emphasis throughout this section on linking imaging data to behavioural factors such as episodic memory, executive control, planning, autobiographical memory, and motor control. This multidisciplinary approach is particularly informative for health professionals working in the field as it provides a clear link between modern scientific methods and clinical diagnosis and symptomatology.

Historically, neuropsychological approaches have been critical to developing knowledge of the frontal lobes and executive function. While advances in neuroimaging have sometimes been argued to have diminished the specialist role of neuropsychology, the fourth section of the text high- lights the ongoing role for neuropsychology, and describes a range of innovative neuropsychological approaches to studying the frontal lobes and their function. An initial chapter on bedside testing illustrates the need for flexible assessment models for 'testing the frontal lobes', while still maintaining the need for standardized and reliable tools. Indeed, it has commonly been noted in the clinical literature that standardized clinical assessments may serve to mask the executive dysfunction that is characteristic of frontal lobe pathology. Stuss' contribution takes this potential limitation on board and describes a set of functional domains (executive cognition, behavioural-emotional self-regulation, energizing regulation and metacognition) that serve to operationalize the behaviours subsumed by the frontal functions into a series of unique components, each argued to have a distinct neuroanatomical substrate. Other chapters focus on specific domains relevant to the frontal lobes including language, social cognition (a new chapter in this edition), selfrepresentation, insight and decision making.

The final two sections of the text are primarily clinically driven, and describe a series of clinical conditions in which frontal functions have been noted to be impaired. Neurological diseases described include primarily degenerative conditions (e.g., frontotemporal dementia, Parkinson's Disease, Lewy Body Dementia, variants of Alzheimer's Disease), as well as a handful of other conditions such as traumatic brain injury and vascular disease. Psychiatric disorders linked to frontal lobe impairment are also canvassed and include depression, bipolar disorder, schizophrenia and obsessive-compulsive disorder. The pattern of each of these chapters is to provide the pathological data for each condition and then link this to behavioural characteristics, most particularly executive functions. A rather puzzling addition to the text, is a chapter on frontal lobe development in childhood. Interestingly, this chapter is located together with the chapters on neurological disorders and disease, but might have been better placed earlier in the text.

In conclusion, this revised text has successfully managed to capture the significant advances since original publication almost a decade ago. It has adopted a strongly evidencebased model to inform the reader of key concepts in the neurosciences and their relationship to clinical practice. This emphasis provides an excellent illustration of the benefits of the scientist-practitioner model. As such, I believe it will be of particular interest to clinicians and health professionals working with adults with neurological and psychiatric conditions. 